\title{
Optimal Distributed Generator Allocation Method Considering Voltage Control Cost
}

\author{
Tie Chen ${ }^{1}$ and Nan Wang ${ }^{2}$ \\ ${ }^{1}$ Harbin Power Supply Company of State Grid Heilongjiang Electric Power Supply Company, Harbin, Heilongjiang, China \\ ${ }^{2}$ School of Electrical Engineering, Northeast Electric Power University, Jilin, Jilin, China \\ ${ }^{*}$ Corresponding author
}

\begin{abstract}
High penetration of intermittent distributed generation access to the grid is a new challenge of the grid planning and operation. In order to achieve the best balance between economic cost and distributed generation capacity, a new method of active distribution network integration considering voltage control cost and voltage control effect is proposed. Firstly, the paper analyzes the voltage regulation characteristics of distributed and centralized voltage regulation, and establishes the technical framework, voltage regulation strategy and economic cost model of different voltage regulation systems. Then, an integrated planning model with objectives of minimizing comprehensive cost and maximizing clean energy utilization under constraints of maintaining voltage acceptable and total cost reasonable was implemented. The simulation results demonstrate that the proposed approach is able to integrate larger distributed generators while maintaining voltage within statutory limits and decrease distribution network operators' economic cost.
\end{abstract}

Keywords-distributed generation; voltage control; active distribution network; integrated planning

\section{INTRODUCTION}

The use of clean renewable energy power generation of distributed wind generation (DWG), photovoltaic (PV) and run-off small hydropower and other intermittent distributed generation (DG) access to the distribution network can save energy, reduce system loss and improve power supply reliability and flexibility [1-2]. However, intermittent DG output is affected by the environment and climate, with obvious intermittency, uncertainty and fluctuation, will affect the normal operation of power system, and its degree of influence is closely related to the siting and sizing of intermittent DG [3]. With the uncertain change of DG and load, the current is bidirectional flowing, the voltage fluctuation is increased, and the power quality problem is especially prominent. Among them, the voltage problem has become an important factor to suppress a large amount of DG access to the grid.

In order to improve the negative impact of DG access to the grid, a variety of solutions are proposed by researchers. Among them, the microgrid through self-control, protection and management achieve DG and load access to the grid, but due to the device is relatively complex, high cost, it's difficult to achieve large-scale popularization [4]. However, ADS planning with flexible grid structures and integrated control systems is much more complex than traditional distribution networks. With the rapid development of communication and Internet technology, control theory and intelligent algorithms are increasingly updated, the concept of cyber physical system (CPS) extends to the power system [5]. Different control methods, scheduling strategies and realization means have different control capabilities, and the complexity of the control system, the support system (communication and automation) needs are closely related to the system cost. Therefore, in the planning and operation of the power network need to consider the impact of secondary systems such as control systems: in the planning stage to consider the control systems cost, control effects and coordination between load and power; in the simulation calculation to consider the voltage control capability, which can improve the planning accuracy, given a reasonable DG interconnection program [6].

According to the above problems, this paper presents a new method of optimal allocation of DG capacity considering the cost and effect of voltage control. This method makes full use of control ability of DG to realize the short-time on-line voltage regulation, with the lowest comprehensive cost and the maximum proportion of the clean energy generation for multiobjective, using the multi-objective differential evolution algorithm to solve problems, so as to ensure that the optimal capacity of DG can meet the requirements of operation state in actual operations, and achieve the optimal planning object [7].

\section{Distribution Network Voltage CONTROL System}

\section{A. Distributed Voltage Control System.}

Distributed voltage control utilizes local information, through the voltage of independent controlling monitoring point to improve the overall operation of the grid, all the voltage and VQC devices are operated locally [8]. Distributed voltage regulation is a local autonomous voltage regulation strategy, so it can overcome the communication line fault and rapid voltage changes when the slow response problems. Secondly, distributed control reduces the investments and costs in communication systems, and it is suitable for operating voltage regulation in the remote areas.

Since only local information is required, the system consists of a local autonomous controller and a corresponding communication device. The economical analysis of the distributed voltage control system includes the cost of the local autonomous controller and the corresponding communication system. The mathematical expression is: 


$$
\begin{gathered}
C_{\mathrm{vo} . \mathrm{de}}=C_{\mathrm{local}}+C_{\mathrm{tx}} \\
C_{\mathrm{tx}}=\sum_{n=1}^{5} \lambda_{n} \beta_{n}+C_{0}
\end{gathered}
$$

Where $C_{\text {vo.de }}$ is the cost of building a distributed voltage control; $C_{\text {local }}$ is the cost of local autonomous controller; $C_{\mathrm{tx}}$ is the cost of building a communication system; $\lambda_{1}, \ldots, \lambda_{5}$ are the length of the communication system fiber laying, EPON-OLT, EPONONU, GPRS terminal and the number of integrated network management equipment respectively; $\beta_{1}, \ldots, \beta_{5}$ are the integrated unit price of the communication system fiber, EPON-OLT, EPON-ONU, GPRS terminal and integrated network management equipment respectively; $C_{0}$ is the construction management fee.

Generally, in order to achieve DG maximum active output, maximize the economic benefits, the generator power factor as close as possible to the rated value, but this measure greatly wastes the DG's own regulator ability. This paper proposes to let DG take the initiative to participate in the distribution network voltage control, grid connected DG is the power factor control, then DG active power output and reactive power output ratio is set to a certain value, the reactive power varies with the change of active power. The generator node in the power flow calculation is the PQ node.

Considering the integrity of the system, this paper has added day-ahead optimization into the distributed voltage control system, using different time scale for coorperation control. Because the day-ahead control and real-time control of this system can be realized without communication, no communication system is needed. The concrete steps are as follows:

1) Day-ahead optimal scheduling: According to the load forecasting, DG output prediction is planned for the distribution network voltage control equipment, and obtain the action sequence of OLTC and capacitor group from the optimization results. Day-ahead optimization can also use the typical day-related equipment action program.

2) Real-time distributed voltage regulator: The initial power factor of each DG is 1 . According to the actual DG output and load fluctuation, through power system flow calculation we can obtain the real-time voltage value. If the monitoring point voltage is over limit at a certain time, starting the voltage regulator system: DG power factor will be decreased because of $\Delta \varphi$ step size, this time the unit will be into the phase running to absorb reactive power and reduce the monitoring point voltage until the power factor can be achieved the adjustable extreme value $\cos \varphi_{\min }$, then regulate the next DG, during the regulating period, once the voltage back to the qualified range, then stop regulating, which means that the regulator strategy regulates successfully.

If the power factor of the last power station reaches $\cos \varphi \min$ but the monitoring point voltage is still unqualified, then stopping the voltage regulation operation. This indicates that the regulating ability of the voltage regulator strategy is limited and the voltage can not be restored to normal level.

\section{B. Centralized Voltage Control System}

Centralized voltage control is the most direct way to manage and optimize the entire distribution network voltage. Through DG and parallel capacitor in distribution network to provide active and reactive power centralized scheduling, constituting the distribution network voltage centralized control, making arrangements and adjustments for the system operation from the perspective of the whole network. However, this control strategy to the network topology information, each node state variables, DG output and other mass data unified processing led to the calculation tends to be complicated, although the use of intelligent algorithms can optimize the speed of solution, but when applied to $10 \mathrm{kV}$ medium voltage distribution network with a wide range and complex equipment, its real-time are still considerable limitations. And in practical applications, centralized control of the communication channel reliability and real-time requirements are very high, and it need sensors, communications equipment, controllers and other investment, which increase the difficulty and cost of control.

The centralized voltage control system consists of three parts: the active distribution network management system (ADMS), the regional coordination controller and the local autonomous controller. In the economic analysis, the construction cost includes ADMS cost, the regional coordination controller cost, the local autonomous controller cost and the corresponding communication system cost. The expression is

$$
C_{\mathrm{vo.cc}}=C_{\mathrm{ADMS}}+C_{\mathrm{area}}+C_{\mathrm{local}}+C_{\mathrm{tx}}
$$

Where $C_{\text {vo.cc }}$ is the centralized voltage control system cost; $C_{\mathrm{ADMS}}$ is the active distribution network management system cost; $C_{\text {area }}$ is the regional coordination controller cost.

The time interval for real-time centralized control is 10 to 15 minutes, so the distribution network needs to have a certain data processing abilities. From the mathematical point of view, the use of optimized ADS centralized reactive voltage control is a multi-objective nonlinear programming problem.

$$
\left\{\begin{array}{l}
\min f\left(x, u_{\mathrm{d}}, u_{\mathrm{c}}\right) \\
g\left(x, u_{\mathrm{d}}, u_{\mathrm{c}}\right)=0 \\
h\left(x, u_{\mathrm{d}}, u_{\mathrm{c}}\right) \leq 0
\end{array}\right.
$$

Where $u_{\mathrm{d}}$ is a discrete control variable, which is usually for switching control variables such as tap position, parallel capacitors and reactors, etc.; $x$ is the dependent variable (only indirect control), which is usually used as the voltage and phase angle of each node of the distribution network; $u_{\mathrm{c}}$ is a continuous control variable, which is usually the active and reactive power of DG in the distribution network. Such as internal point method, the objective function is: 


$$
\min f\left(x, u_{\mathrm{d}}, u_{\mathrm{c}}\right)=\sum_{i=1}^{N}\left[P_{\mathrm{L}}+\lambda \sum_{j=1}^{n}\left(\frac{\Delta V_{j}}{V_{j \max }-V_{j \min }}\right)^{2}\right]
$$

\section{OPTIMIZATION OF DG CAPACITY BASED ON VOLTAGE REGULATION}

\section{A. Multi-objective Differential Evolution Algorithm}

The capacity optimization model of DG is a multi-objective optimization problem which includes two objective functions: the minimum annual comprehensive cost and the maximum proportion of clean energy generation. In the solution of multiobjective optimization problem, it is necessary to satisfy two or more objectives at the same time, but these objectives may be contrary to each other. Therefore, in the solution of multiobjective optimization problem, we usually use compromise solution set, that is, Pareto solution set [9]. Researchers find a new intelligent optimization algorithm based on population optimization, namely, differential evolution (DE). DE has many characteristics such as rapidly convergence rate, few adjustable parameters and good robustness [10].

Similar to the normal differential evolution, multi-objective differential evolution is also completed by some steps such as population initialization, crossover, mutation and selection [11]. However, different from differential evolution with only choosing objective function values, the evolution of population with multi-objective differential evolution is based on rapid non-dominated sorting and congestion calculation.

\section{B. Optimization process based on multi-objective differential evolution algorithm}

The specific steps for optimizing the DG access capacity with the voltage regulation are as follows.

Input network parameters, parameter initialization and population initialization for mutation operation and crossover operation [11].

According to the load forecast, predicted output data of DG, we determine the day-ahead voltage regulation program [12]. According to the actual load, actual output data of DG, we make DG participate in real-time voltage regulation.

The multi-objective optimization problem includes two objective functions: the minimum annual comprehensive cost and the maximum proportion of clean energy generation, and its constraint condition is voltage quatification rate [13]. Then we can obtain the optimal compromise solution through the intelligent algorithm, and select the most suitable DG access capibility and voltage regulation strategies.

\section{Example Simulation}

In this paper, we take IEEE-33 node distribution system as an example. The distribution system has 33 nodes, where node 1 is the root node which is connected with the infinite system. Small hydropower in clean energy has a wide range of distribution, flexible power supply and other characteristics.
However, because of the small mountainous load, a large number of small hydropower access to power grid often lead to high voltage, greatly limiting the access capacity of small hydropower [14]. Therefore, aiming at the above problems, this paper's method not only provides solving thoughts to the problem, but also for a large number of photovoltaic, wind power access to grid planning issues put forward a technical support [15]. According to the actual installation of small hydropower stations, the distribution system at the end of each feeder network access to four small hydropower stations, as shown in Figure 3. In the differential evolution algorithm of this paper, the population size is 100 , the maximum number of iterations is 2000 , the maximum and minimum scale factors are 0.9 and 0.1 respectively, and the maximum and minimum cross factors are 0.8 and 0.3 respectively.



FIGURE I. 33-BUS DISTRIBUTION SYSTEM WITH SHP.

During the simulation process, the day-ahead voltage regulation scheme adopts the conventional typical day's action parameters, and the initial power factor of the small hydropower is 1.0 in the real-time regulation. Considering the accuracy and computational complexity, we use the operation condition of the typical day to represent the operation of the whole year [6].

\section{A. Simulation Analysis of Multi-objective Optimal Access Capacity}

Because the small hydropower is distributed in the remote mountainous area, the laying of the wired communication lines are difficult, the stability of the wireless communication is poor , the building cost of the communication system is seem as infinite, and the centralized voltage coordination control of the global information can't be realized. So instead of the centralized voltage coordination control, we use distributed voltage coordinated control of the non-communication system, and build DG to participate in the distributed voltage regulation system.

After the voltage regulation strategy is determined, the multi-objective optimization problem simplified to two objective functions: the minimum annual comprehensive cost and the maximum proportion of clean energy generation, and the Pareto solution is obtained by the multi-objective differential evolution algorithm, as shown in figure 2 .

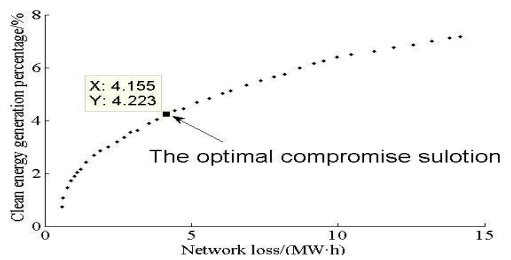

FIGURE II. PARETO FRONT SOLUTION SET. 
In the acceptable range of the total network loss, we select the optimal compromise solution. The optimal capacity of the multi-objective differential evolution algorithm is $7.6553 \mathrm{MW}$ nodes $18,22,25$ and 33 are $0.6846,2.7745,2.9072$ and 1.289 MW respectively. At this time, the voltage qualification rate of the typical day's monitoring time of the distribution network is 1. The network loss per day is $4.2536 \mathrm{MW} \cdot \mathrm{h}$, the network loss rate is $3.26 \%$, the proportion of clean energy generation is $4.2484 \%$, the power reversing situation occurs, and the voltage regulation system is involved in the regulation operation.

\section{B. Effect of Voltage Regulating System on DG Permeability}

If the voltage regulation ability of the distribution network is not taken into account, we can use the multi-objective differential evolution algorithm to solve the optimal access capacity of small hydropower. The optimal total capacity of small hydropower is $6.782 \mathrm{MW}$, and nodes $18,22,25$ and 33 are $0.5941,2.3420,2.8134$ and 1.0325 MW respectively, the network loss per day is $3.3473 \mathrm{MW} \cdot \mathrm{h}$, the network loss rate is $1.52 \%$, and the proportion of clean energy generation is $3.7637 \%$.

By the simulation results can be drawn, the voltage regulation system due to the effectively improve the network voltage problems after a large number of DG access to the gird, and increase the ADS consumptive ability to DG. Although the network loss has slightly increased, it is still within the acceptable range.

\section{CONCLUSION}

This paper presents an ADS planning method to improve the efficiency of clean energy utilization and reduce the comprehensive cost of grid companies under the premise of ensuring the qualified voltage. We establish the optimization model of small hydropower access capacity with voltage control capability. The planning objectives are the smallest annual comprehensive cost and the largest proportion of clean energy, and the constraint condition is the qualified voltage, then we use the IEEE 33-node distribution system to study simulation analysis. Based on the above theoretical analysis and simulation results, the conclusions are drawn as follows:

The cost of the secondary system (control system and its supporting system) is taken into account in the planning objectives. Therefore, the influence of the control strategy on the operation cost and the construction cost is taken into account. The simulation process takes into account the regulation effect, so it can visually evaluate the regulation system control ability. Considering the above two aspects, we can get a more comprehensive DG optimization scheme, which improves the accuracy of ADS planning after a large number of DG access to the grid.

Different control systems have different effects on grid planning and operation. Distributed and centralized voltage control can both alleviate the problem of voltage rise and improve DG permeability to a certain extent, and the latter is better. But centralized control means that the relevant cost of investment is greater. As the grid becomes more intelligent, automated and complex, the cost of these systems' proportion of total cost is increasing. Therefore, if we first consider the maximum benefits, then select the ditributed model; if we first conside the control effect and DG permeability, then select the centralized model.

\section{REFERENCES}

[1] Kang Longyun, Guo Hongxia, Wu Jie, et al. Characteristics of distributed generation system and related research issues caused by connecting it to power system. Power System Technology, 2010, vol. 34, pp. 43-47(in Chinese).

[2] Pei Wei, Peng Kun, distributed generation Kong Li, et al. Impact and improvement of distributed generation on distribution network voltage quality. Power System Technology, 2008, vol. 28, pp. 152-157 (in Chinese).

[3] Kashem M A, Le A D T, Negnevitsky M, et al. Distributed generation for minimization of power losses in distribution systems, IEEE Power Engineering Society General Meeting. Montreal, Canada: IEEE, 2006, pp. 1-8.

[4] A1Kaabi Sultan S, Zeineldin H H, Vinod Khadkikar. Planning active distribution networks considering multi-DG configurations. IEEE Transactions on Power Systems, 2014, vol. 29, pp. 785-793.

[5] Kiprakis A E, Wallace A R. Maximising energy capture from distributed generators in weak networks. IEEE Proc. on Generation, Transmission and Distribution, 2004, vol. 151, pp. 611-618.

[6] Eberly T W, Schaefer R C. Voltage versus var/power-factor regulation on synchronous generators. IEEE Transactions on Ind. Appl., 2002, vol. 38, pp. 1682-1687.

[7] MARANO A, ORTEGA J M M, RAMOSJ L M, et al. Voltage control of active distribution networks by means of dispersed generation. Integration of Renewables into the Distribution Grid, CIRED2012 Workshop. Lisbon, Portugal: IET, 2012, pp. 1-4.

[8] Chen Fei, Liu Dong, control strategy for Chen Yunhui. Hierarchically distributed voltage active distribution network. Automation of Electric Power Systems, 2015, vol. 39, pp. 61-67 (in Chinese).

[9] Hatta H, Kobayashi H. A study of centralized voltage control method for distribution system with distributed generation. $19^{\text {th }}$ International Conference on Electricity Distribution (CIRED). Vienna: CIRED, 2007, pp. 330 .

[10] Hashim T J T, Mohamed A, Shareef H. A review on voltage control methods for active distribution networks. PRZEGLD ELEKTROTECHMCZNY (Electrical Review), 2012, pp. 304-312

[11] Conti S, Greco A M. Active MV distribution network planning coordinated with advanced centralized voltage regulation system. Power Tech, 2007 IEEE Lausanne. Lausanne: IEEE, 2007, pp. 2105-2109.

[12] Sansawatt T, O'Donnell J, Ochoa L F, et al. Decentralised voltage control for active distribution networks. Universities Power Engineering Conference (UPEC), 2009 Proceedings of the $44^{\text {th }}$ International. Glasgow: IEEE, 2009, pp. 1-5.

[13] Vows P N, Kiprakis A E, Wallace A R, et al. Centralized and distributed voltage control:impact on distributed generation penetration. IEEE Transactions on Power Systems, 2007, vol. 22, pp. 476-483.

[14] Zhou Y, Li X, Gao L. A differential evolution algorithm with intersectmutation operator. Applied Soft Computing, 2013, vol. 13, pp. 390-401.

[15] Yan Hongwen, Li Ximan. Uncertainty analysis on net load forecasting for busbar containing distributed energy sources based on differential evolution and rough sets reduction. Power System Technology, 2013, vol. 37, pp. 1602-1606 (in Chinese). 